\title{
Association between adherence to medications for COPD and medications for other chronic conditions in COPD patients
}

This article was published in the following Dove Press journal:

International Journal of COPD

22 December 2016

Number of times this article has been viewed

\author{
Amol D Dhamane' \\ Phil Schwab ${ }^{2}$ \\ Sari Hopson ${ }^{2}$ \\ Chad Moretz ${ }^{2}$ \\ Srinivas Annavarapu ${ }^{2}$ \\ Kate Burslem' \\ Andrew Renda ${ }^{3}$ \\ Shuchita Kaila' \\ 'Health Economics and Outcomes \\ Research, Boehringer Ingelheim \\ Pharmaceuticals Inc, Ridgefield, CT, \\ ${ }^{2}$ Comprehensive Health Insights Inc, \\ Louisville, ${ }^{3}$ Humana Inc, Louisville, \\ KY, USA
}

Correspondence: Phil Schwab Comprehensive Health Insights Inc, 5 I 5 W Market St., Louisville, KY 40202, USA

Tel +I 5024765610

Email pschwab@humana.com
Background: Patients with COPD often have multiple comorbidities requiring use of multiple medications, and adherence rates for maintenance COPD (mCOPD) medications are already known to be suboptimal. Presence of comorbidities in COPD patients, and use of medications used to treat those comorbidities (non-COPD medications), may have an adverse impact on adherence to mCOPD medications.

Objective: The objective of the study was to evaluate the association between non-adherence to mCOPD medications and non-COPD medications in COPD patients.

Methods: COPD patients were identified using a large administrative claims database. Selected patients were 40-89 years old and continuously enrolled for 12 months prior to and 24 months after the first identified COPD diagnosis (index date) during January 1, 2009 to December 31, 2010. Patients were required to have $\geq 1$ prescription for a mCOPD medication within 365 days of the index date and $\geq 1$ prescription for one of 12 non-COPD medication classes within \pm 30 days of the first COPD prescription. Adherence (proportion of days covered [PDC]) was measured during 365 days following the first COPD prescription. The association between non-adherence (PDC $<0.8)$ to $\mathrm{mCOPD}$ and non-adherence to non-COPD medications was determined using logistic regression, controlling for baseline patient characteristics.

Results: A total of 14,117 patients, with a mean age of 69.9 years, met study criteria. Of these, $40.9 \%$ were males and $79.2 \%$ were non-adherent to mCOPD medications with a mean PDC of 0.47 . Non-adherence to $\mathrm{mCOPD}$ medications was associated with non-adherence to 10 of 12 non-COPD medication classes (odds ratio 1.38-1.78, all $P<0.01$ ).

Conclusion: Adherence to mCOPD medications is low. Non-adherence (or adherence) to mCOPD medications is positively related to non-adherence (or adherence) to non-COPD medications, implying that the need to take medications prescribed for comorbid conditions does not adversely impact adherence to MCOPD medications.

Keywords: COPD, comorbidities, adherence, PDC, database, Medicare

\section{Introduction}

Chronic lower respiratory diseases are the third leading cause of death in the US. ${ }^{1}$ One of the most prevalent diseases in this category is COPD, which is characterized by persistent airflow limitation. ${ }^{2}$ In the US, COPD affects approximately 24 million adults. ${ }^{3}$ COPD is a progressive disease, with typical symptoms including sputum production, cough, and chronic and progressive dyspnea. ${ }^{2}$ Exacerbations, the sudden worsening of typical COPD symptoms, often complicate COPD, and are associated with reduction in health-related quality of life, declines in lung function, and increased hospitalizations and mortality. ${ }^{4}$ 
Although the long-term decline of lung function in COPD patients cannot be reversed by pharmacotherapy, medications are available to prevent and control symptoms, improve health status, and reduce the occurrence of COPD exacerbations. ${ }^{2}$ Medication adherence levels for COPD medications have been found to be well below the levels typically observed for treatments for other conditions. ${ }^{5-8}$ For example, in a 2011 study of US military veterans, proportion of patients adherent to COPD treatments, defined as proportion of days covered (PDC) $\geq 0.80$, was reported to be approximately $30 \%$ versus $40 \%-63 \%$ for coronary artery diseases, diabetes, heart failure, and hyperlipidemia treatments. ${ }^{8}$

One potential reason for lower adherence to COPD medications may be the daily variations in symptoms experienced by COPD patients. Symptoms specific to COPD, such as breathlessness, cough, and sputum production, may fluctuate daily. Physicians typically prescribe rescue medications to alleviate these symptoms, and advise their patients to adjust their rescue medication use according to the symptoms experienced on any given day., ${ }^{2,9}$ Additionally, physicians often prescribe these rescue medications in conjunction with COPD maintenance medications depending on severity of the disease. Instructions to use or not use rescue medications based on symptoms while also managing maintenance medications that are often available in different delivery devices may result in patient confusion. This complexity could ultimately lead patients to deviate from their prescribed dosing regimen of COPD maintenance medications.

Furthermore, COPD patients typically have multiple chronic comorbidities including conditions such as hypertension, coronary atherosclerosis, other heart diseases, lipid disorders, diabetes, osteoporosis, and sleep apnea. ${ }^{10,11}$ In one study, $78 \%$ of participants with COPD had one or more comorbidities, compared with about $50 \%$ of participants without COPD. ${ }^{12}$ It is expected that COPD patients are prescribed several concurrent therapies for these comorbidities, and COPD medication adherence may be impacted by this comorbidity burden and the correspondingly complex medication regimen. Other common reasons for non-adherence to medications in general include health system factors such as medication reimbursements and time spent with physicians, socioeconomic factors such as income and transportation, and patient-related factors such as knowledge and beliefs. ${ }^{13}$

In this study, we sought to describe and assess the association between adherence to maintenance COPD (mCOPD) medications and adherence to medications used to treat chronic conditions other than COPD (non-COPD medications). Understanding the relationship may provide further insight into the reasons for suboptimal adherence rates for COPD medications.

\section{Methods \\ Design and data source}

This observational study utilized retrospective administrative claims data from the Humana Research Database (Louisville, KY, USA) from January 1, 2008 to December 31, 2012 (study period). Data used for the study included inpatient and outpatient medical and pharmacy claims as well as enrollment information for members participating in commercial insurance plans and Medicare Advantage plans with Prescription Drug benefits (MAPD). The study protocol, waiver of written informed consent, and waiver of authorization to use and disclose protected health information were approved by Schulman IRB. (Cincinnati, OH, USA), an independent Institutional Review Board (IRB) fully accredited by the Association for the Accreditation of Human Research Protection Programs (registration number is 00000971). This study followed the principles outlined in the Declaration of Helsinki.

\section{Patient selection}

Patients included in this study population were required to have a COPD diagnosis and were also required to initiate $\mathrm{mCOPD}$ medication within a year of COPD diagnosis. A COPD diagnosis was determined by presence of two or more medical claims with a COPD diagnosis code (International Classification of Diseases, Ninth Revision, Clinical Modification [ICD-9-CM] code: 491.xx [chronic bronchitis], 492.xx [emphysema], or 496.xx [COPD, unspecified]) in primary or secondary position during the identification period of January 1, 2009 to December 31, 2010. The two medical claims with a COPD diagnosis code had to occur on separate days, occurring no more than 90 days apart. The earliest date of the medical claim with a COPD diagnosis code served as the index date. Patients were required to be continuously enrolled in a commercial or MAPD plan for a 12-month period prior to the index date (pre-index period) and a 24-month period following index date (post-index period) and were required to be between the age of 40 and 89 years on the index date.

To assess initiation of mCOPD medication therapy within a year of COPD diagnosis, patients were required to have at least one prescription claim for a $\mathrm{MCOPD}$ medication (most frequently prescribed classes of mCOPD medications during the study identification period: long-acting muscarinic antagonists [LAMA] and inhaled corticosteroidlong-acting beta agonist [LABA] fixed-dose combinations, 
Table I GPI codes for maintenance COPD medications and non-COPD medications

\begin{tabular}{ll}
\hline Drug/drug class name & GPI code \\
\hline mCOPD medications & \\
Aclidinium & $4410000710^{*}$ \\
Tiotropium & $4410008010^{*}$ \\
Budesonide-formoterol & $442099024 I^{*}$ \\
Fluticasone-salmeterol & $4420990270^{*}$ \\
Mometasone-formoterol & $4420990290^{*}$ \\
Non-COPD medications & \\
Antianxiety & $57^{*}$ \\
Anticoagulants & $8310^{*}, 8320^{*}, 8333^{*}, 8337^{*}$ \\
Antidepressants & $58^{*}$ \\
Antihyperglycemics & $2715^{*}, 2717^{*}, 2720^{*}, 2725^{*}, 2728^{*}$, \\
& $2750^{*}, 2755^{*}, 2757^{*}, 2760^{*}, 2799^{*}$ \\
Antihypertensives & $36^{*}$ \\
Beta-blockers & $33^{*}$ \\
Bisphosphonates & $300420^{*}$ \\
Calcium channel blockers & $34^{*}$ \\
Diuretics & $37^{*}$ \\
Insulin & $2710^{*}$ \\
NSAIDs & $6610^{*}$ \\
Statins & $3940^{*}$ \\
\hline Note:Al
\end{tabular}

Note: *All medications with the GPI prefix were included for identification. Abbreviations: GPI, generic product identifier; mCOPD, maintenance COPD; NSAIDs, nonsteroidal anti-inflammatory drugs.

Table 1) during the first 365 days of the 24 -month postindex period. This criterion allowed for the assessment of 1 -year mCOPD medication adherence in the post-index period. The date of the first prescription claim for a mCOPD medication was termed the MCOPD medication start date. In addition, to assess initiation of non-COPD medication (Table 1, medications use to treat chronic conditions other than COPD), patients were required to have at least one prescription claim for a non-COPD medication and this claim had to occur within 30 days of the MCOPD medication start date.

Patients were excluded from the study if they had $\geq 1$ medical claim with any of the following ICD-9-CM diagnosis codes in any position during the study period: $277.0 \mathrm{x}$ (cystic fibrosis), 011.x (pulmonary tuberculosis), or 140.xx-172.xx, 174.xx-209.3x, 209.7x (malignant neoplasms).

\section{Adherence to mCOPD medications}

Adherence to mCOPD medications was the main outcome variable of this study. Adherence was measured in terms of the PDC. PDC was measured over a 365-day period following the $\mathrm{mCOPD}$ medication start date. PDC for $\mathrm{mCOPD}$ medications was defined as the sum of the number of nonoverlapping days-supply of prescriptions for mCOPD medications, divided by 365 . No credit was given for overlap of days-supply. ${ }^{14}$ Patients were deemed adherent to mCOPD medications if the PDC was greater than or equal to the 0.80 threshold and non-adherent otherwise.

\section{Adherence to non-COPD medications}

Adherence to non-COPD medications was hypothesized to have an impact on adherence to mCOPD medications. This was also assessed using PDC..$^{14}$ It was measured from the date of the first prescription for a non-COPD medication and ended at 364 days following the mCOPD medication start date. PDC for non-COPD medications was defined as the sum of the number of nonoverlapping days-supply of prescriptions for non-COPD medications, divided by 365 . No credit was given for overlap of days-supply. ${ }^{14}$ Patients were deemed adherent to non-COPD medications if the PDC was greater than or equal to the 0.80 threshold and non-adherent otherwise.

Adherence to each of the following 12 non-COPD medication classes were measured: statins, antihypertensives, anticoagulants, oral antihyperglycemics, insulins, antidepressants, nonsteroidal anti-inflammatory medications (NSAIDs), antianxiety, calcium channel blockers, beta-blockers, bisphosphonates, and diuretics (Table 1). These 12 non-COPD medication classes were identified based on the medications prescribed for the most common comorbidities observed in the selected patient population.

\section{Baseline patient characteristics}

Baseline patient characteristics were measured to describe the study population and included age (as of index date), sex, and geographic location (as of index date) and influenza vaccination status. Influenza vaccination status was defined as null, partial, or full based on the number of pharmacy or medical claims for influenza vaccines and the timing of these claims during the 24-month post-index period as follows: null = zero pharmacy or medical claims indicating an influenza vaccine during the post-index period; partial = one pharmacy or medical claim indicating an influenza vaccine during the post-index period; full = at least two pharmacy and/or medical claims indicating an influenza vaccine and occurring at least 6 months apart during the post-index period. General comorbidity burden was assessed using the RxRisk-V score. The RxRisk-V score is a prescription claim-based comorbidity index. This score involves the creation of 45 indicators for general drug categories (eg, human immunodeficiency virus drugs, diabetes drugs) and durable medical equipment (DME) categories. These indicator variables are summed to create the total RxRisk-V score. For this study, the DME categories were not included for the RxRisk- $\mathrm{V}$ calculation. 
Only the 42 drug categories were included. The RxRisk-V has been rigorously tested and has demonstrated concordance with the Deyo-Charlson comorbidity index score. ${ }^{15}$

\section{Statistical analysis}

Summary statistics including mean, median, and interquartile range were provided for adherence to $\mathrm{mCOPD}$ and nonCOPD medications. Summary statistics for baseline patient characteristics were also reported for the study population and compared between patients adherent ( $\mathrm{PDC} \geq 0.8$ ) and nonadherent (PDC $<0.8$ ) to mCOPD medications. Unadjusted, statistical comparisons of baseline patient characteristics were made between patients adherent (PDC $\geq 0.8$ ) and non-adherent $(\mathrm{PDC}<0.8)$ to $\mathrm{mCOPD}$ medications using chi-square and non-parametric Wilcoxon Mann-Whitney (rank sum) test for categorical and continuous variables, respectively.

Association between adherence to $\mathrm{mCOPD}$ and nonCOPD medications was assessed using a logistic regression model controlling for baseline patient characteristics including age, sex, region, influenza vaccination status, and RxRisk-V score. The dependent variable for this model was non-adherence (PDC <0.8) to mCOPD medications, and the main independent variables of this model were non-adherence (PDC <0.8) to each of the 12 non-COPD medication classes. Odds ratios along with $95 \%$ confidence intervals were reported.

\section{Results}

The study population consisted of a total of 14,117 COPD patients that met all study criteria (Figure 1) with a mean age of 69.9 years; $40.9 \%$ of the patients were males and twothirds of the study population were residing in the southern US. The mean RxRisk-V score was 7.2, and $38.9 \%$ of the study population did not receive a claims-identifiable influenza vaccination during the post-index period.

On the index date, $32.0 \%$ had a pharmacy claim for a LAMA only, $59.8 \%$ had a claim for an ICS/LABA only, and $8.2 \%$ had claims for LAMA and ICS/LABA. Mean adherence to mCOPD medications was 0.47 , and less than one quarter $(20.8 \%)$ of the study population was deemed adherent based on the threshold of PDC $\geq 0.80$. Baseline patient characteristics stratified by $\mathrm{mCOPD}$ medication adherence status are presented in Table 2 . No statistically significant differences were found between the patients adherent to mCOPD medications and non-adherent to mCOPD medications with respect to sex and age, but there were statistically significant differences with respect to geographical
Patients with a COPD diagnosis ${ }^{a}$ during the identification period: January 1, 2009 to December 31, 2010

Initial study population, $\mathrm{N}=\mathbf{2 5 5 , 9 7 3}$

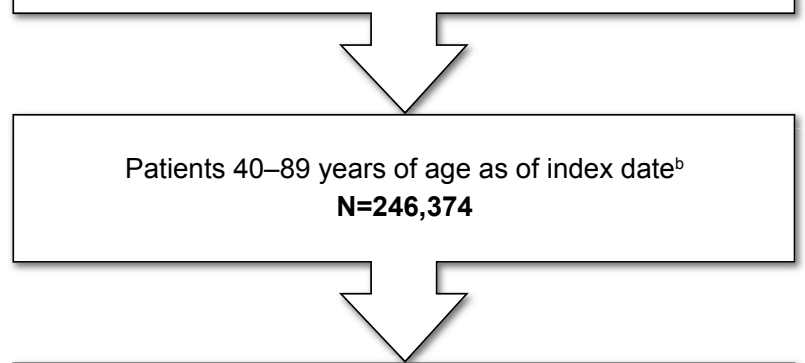

Patients continuously enrolled in a commercial or MAPD plan during the pre-index period ${ }^{c}$ and post-index period ${ }^{d}$ $\mathrm{N}=\mathbf{8 4 , 8 1 1}$

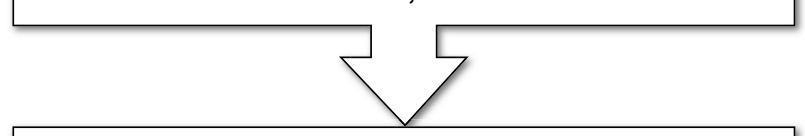

Patients with no cystic fibrosis, pulmonary tuberculosis, or malignant neoplasm diagnosis ${ }^{\mathrm{e}}$ during the study period $\mathrm{N}=52,643$
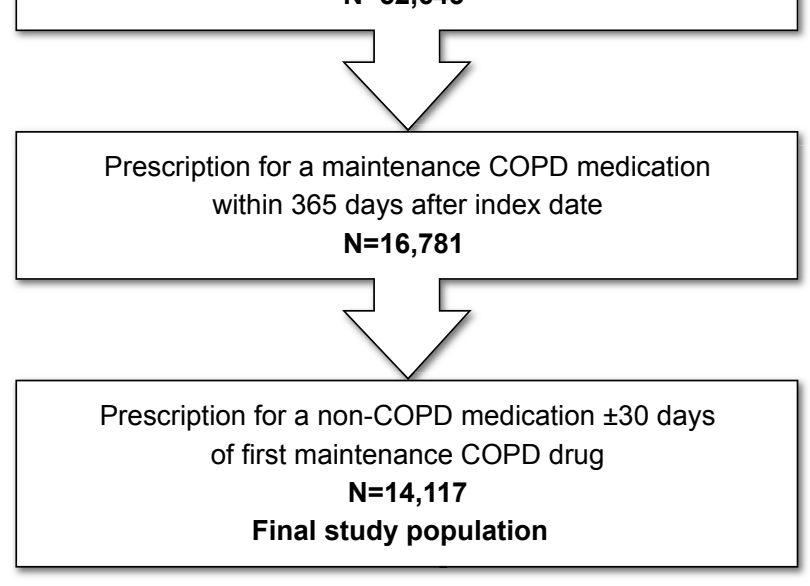

Figure I Patient selection.

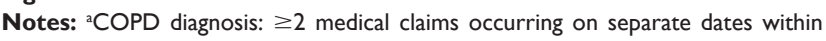
90 days with a COPD diagnosis code (ICD-9-CM code 491.xx, 492.xx, or 496.xx) in the primary or secondary position. IIndex date: The date of first medical claim with a COPD diagnosis during the identification period. 'Pre-index period: A 12-month period prior to index date. ${ }^{\text {P}}$ Post-index period: A 24-month period after index date. ${ }^{e} \geq$ I medical claim with any of the following ICD-9-CM diagnosis codes in any position: 277.0x (cystic fibrosis), 01 I.x (pulmonary tuberculosis), or 140.xx-172.xx, 174.xx-209.3x, 209.7x (malignant neoplasms).

Abbreviations: ICD-9-CM, International Classification of Diseases, Ninth Revision, Clinical Modification; MAPD, Medicare Advantage plans with Prescription Drug benefits.

location, comorbidity burden, and influenza vaccination status. The non-adherent group was more likely to reside in the south $(67.5 \%$ vs $59.2 \%, P<0.0001)$ and less likely to reside in the northeast $(P=0.0003)$, Midwest $(P<0.0001)$ and west $(P=0.0037)$ compared to the adherent group. The non-adherent group also had a greater comorbidity burden compared to the adherent group (mean RxRisk-V score 7.2 vs 7.0, $P<0.0001$ ), was less likely to have the recommended 
Table 2 Baseline patient characteristics: overall and by mCOPD medication adherence status ${ }^{\mathrm{a}}$

\begin{tabular}{|c|c|c|c|c|}
\hline Baseline patient characteristic & $\begin{array}{l}\text { Overall } \\
(n=|4, I| 7)\end{array}$ & $\begin{array}{l}\text { Adherent to } \\
\text { mCOPD medications } \\
(n=2,940)\end{array}$ & $\begin{array}{l}\text { Non-adherent to } \\
\text { mCOPD medications } \\
(n=I I, 177)\end{array}$ & $P$-value \\
\hline \multicolumn{5}{|l|}{ Age, years } \\
\hline Mean (SD) & $69.9( \pm 9.2)$ & $69.8( \pm 8.8)$ & $70.0( \pm 9.3)$ & 0.3402 \\
\hline Median (IQR) & $70.0(66.0-76.0)$ & $70.0(66.0-76.0)$ & $70.0(66.0-76.0)$ & \\
\hline \multicolumn{5}{|l|}{ Age category, n (\%) } \\
\hline 40-49 years & $369(2.6)$ & $67(2.3)$ & $302(2.7)$ & 0.2008 \\
\hline $50-59$ years & $\mathrm{I}, 47 \mathrm{I}(\mathrm{I} 0.4)$ & $305(10.4)$ & $\mathrm{I}, \mathrm{I} 66(10.4)$ & 0.9270 \\
\hline $60-69$ years & $4,799(34.0)$ & $\mathrm{I}, 022(34.8)$ & $3,777(33.8)$ & 0.3235 \\
\hline 70-79 years & $5,324(37.7)$ & I, I35 (38.6) & $4,189(37.5)$ & 0.2620 \\
\hline $80-89$ years & $2,154(15.3)$ & $4 I I(14.0)$ & $\mathrm{I}, 743(\mathrm{I} 5.6)$ & 0.0303 \\
\hline \multicolumn{5}{|l|}{ Sex, $n(\%)$} \\
\hline Female & $8,348(59.1)$ & $\mathrm{I}, 769(60.2)$ & $6,579(58.9)$ & 0.1992 \\
\hline Male & $5,769(40.9)$ & I,I7I (39.8) & $4,598(4 I . I)$ & \\
\hline \multicolumn{5}{|l|}{ Geographic location, n (\%) } \\
\hline Northeast & $254(1.8)$ & $76(2.6)$ & $178(1.6)$ & 0.0003 \\
\hline Midwest & $3,48 \mathrm{I}(24.7)$ & $858(29.2)$ & $2,623(23.5)$ & $<0.0001$ \\
\hline South & $9,285(65.8)$ & $\mathrm{I}, 740(59.2)$ & $7,545(67.5)$ & $<0.0001$ \\
\hline West & $\mathrm{I}, 097(7.8)$ & $266(9.0)$ & $831(7.4)$ & 0.0037 \\
\hline \multicolumn{5}{|l|}{ Line of business, $\mathbf{n}(\%)$} \\
\hline Medicare & $13,5 \mid 2(95.7)$ & $2,789(94.9)$ & $10,723(95.9)$ & 0.0105 \\
\hline Commercial & $605(4.3)$ & $|5|(5.1)$ & $454(4.1)$ & \\
\hline \multicolumn{5}{|l|}{ RxRisk-V comorbidity score ${ }^{b}$} \\
\hline Mean (SD) & $7.2( \pm 3.1)$ & $7.0( \pm 3.1)$ & $7.2( \pm 3.1)$ & 0.0004 \\
\hline Median (IQR) & $7.0(5.0-9.0)$ & $7.0(5.0-9.0)$ & $7.0(5.0-9.0)$ & \\
\hline \multicolumn{5}{|l|}{ Influenza vaccination status, $\mathbf{n}(\%)$} \\
\hline Nullc & $5,488(38.9)$ & $\mathrm{I}, 042(35.4)$ & $4,446(39.8)$ & $<0.0001$ \\
\hline Partial $^{c}$ & $4,434(31.4)$ & $957(32.6)$ & $3,477(31.1)$ & 0.1338 \\
\hline Fullc & $4,195(29.7)$ & $94 I(32.0)$ & $3,254(29.1)$ & 0.0023 \\
\hline
\end{tabular}

Notes: aMeasured during the 12-month pre-index period. ${ }^{\mathrm{b}} \mathrm{RxR}$ isk-V score without DME ranges from 0 to 42 , with a higher score indicating a greater chronic disease burden. Influenza vaccination status was based on the number and timing of vaccine claims during the post-index period as follows: null $=0$; partial $=\geq \geq$; full $=\geq 2$ claims occurring 6 months or greater apart.

Abbreviations: DME, durable medical equipment; IQR, interquartile range; SD, standard deviation; mCOPD, maintenance COPD.

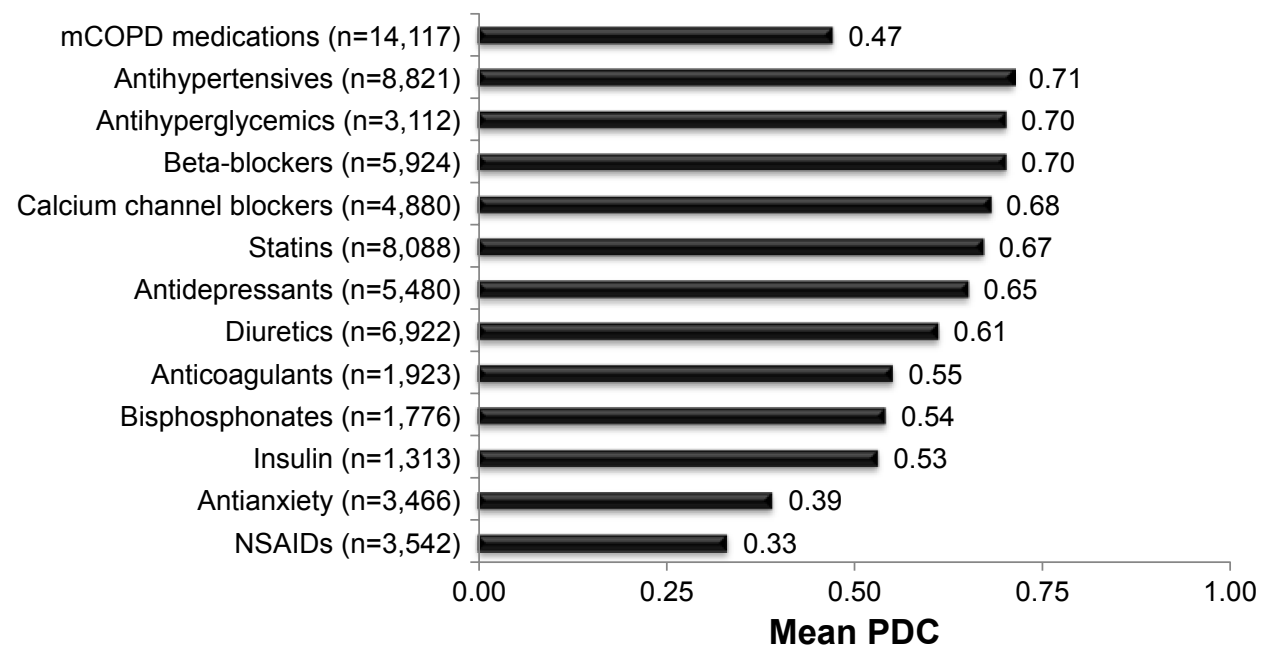

Figure 2 Adherence measured as PDC for $\mathrm{mCOPD}$ and non-COPD medications.

Abbreviations: mCOPD, maintenance COPD; NSAIDs, nonsteroidal anti-inflammatory medications; PDC, proportion of days covered. 
Table 3 Association between non-adherence to mCOPD medications and non-adherence to non-COPD medications: logistic regression analysis ${ }^{\mathrm{a}}$

\begin{tabular}{|c|c|c|c|c|}
\hline Variable & Odds ratio & Lower $95 \% \mathrm{Cl}$ & Upper $95 \%$ Cl & $P$-value \\
\hline \multicolumn{5}{|l|}{ Dependent variable } \\
\hline Non-adherence (PDC $<0.8)$ to $\mathrm{mCOPD}$ medications & NA & NA & NA & NA \\
\hline \multicolumn{5}{|l|}{ Main independent variables } \\
\hline \multicolumn{5}{|l|}{ Non-adherence (PDC $<0.8)$ to } \\
\hline Statins & 1.54 & 1.37 & 1.74 & $<0.0001$ \\
\hline Antihypertensives & 1.55 & 1.38 & 1.74 & $<0.0001$ \\
\hline Anticoagulants & 1.21 & 0.93 & 1.57 & 0.1497 \\
\hline Oral antihyperglycemics & 1.38 & 1.13 & 1.69 & 0.0019 \\
\hline Insulins & 1.78 & 1.28 & 2.47 & 0.0005 \\
\hline Antidepressants & 1.73 & 1.50 & 1.99 & $<0.0001$ \\
\hline NSAIDs & 1.74 & 1.39 & 2.18 & $<0.0001$ \\
\hline Antianxiety & 1.16 & 0.93 & 1.45 & 0.1873 \\
\hline Calcium channel blockers & 1.53 & 1.31 & 1.78 & $<0.0001$ \\
\hline Beta-blockers & 1.39 & 1.21 & 1.61 & $<0.0001$ \\
\hline Bisphosphonates & 1.51 & 1.20 & 1.91 & 0.0005 \\
\hline Diuretics & $\mathrm{I} .44$ & 1.27 & 1.63 & $<0.0001$ \\
\hline
\end{tabular}

Notes: aStudy population, $n=|4|$,17 . Baseline patient characteristics including age, sex, region, influenza vaccination status, and RxRisk-V score were included in the model as covariates.

Abbreviations: $\mathrm{Cl}$, confidence interval; mCOPD, maintenance COPD; NA, not applicable; NSAIDs, nonsteroidal anti-inflammatory drugs; PDC, proportion of days covered.

frequency of influenza vaccinations (influenza vaccination status = full: $29.1 \%$ vs $32.0 \%, P=0.0023)$, and had a higher proportion of patients without any influenza vaccinations during the post-index period compared to the adherent group $(P<0.05)$.

Use of and adherence to the 12 classes of non-COPD medications are presented in Figure 2. Of the 12 classes examined, antihypertensives were the most commonly prescribed (62.5\%) non-COPD medication, while insulin was the least commonly prescribed (9.3\%). Mean PDC for mCOPD medications was 0.47 , whereas mean PDC for the non-COPD medication classes examined ranged from 0.33 to 0.71 .

Multivariate analyses showed that non-adherence to antidepressants, antihypertensives, oral antihyperglycemics, beta-blockers, bisphosphonates, calcium channel blockers, diuretics, insulins, NSAIDs, and statins was each independently associated with increased odds of non-adherence to mCOPD medications (Table 3). Non-adherence to insulins, NSAIDs, and antidepressants had the strongest association with non-adherence to mCOPD medications. Patients non-adherent to insulins, NSAIDs, and antidepressants were $1.78(P<0.01), 1.74(P<0.0001)$, and 1.73 $(P<0.0001)$ times more likely to be non-adherent to mCOPD medications, respectively, after controlling for adherence or non-adherence with other medication classes and controlling for baseline patient characteristics. The model suggested that non-adherence to anticoagulants and antianxiety medications was not associated with non-adherence to mCOPD medications even with consideration of adherence or nonadherence to the other medication classes (Table 3).

\section{Discussion}

COPD is a debilitating disease and is often complicated by exacerbations. ${ }^{4}$ Although several medications are available to prevent and control COPD symptoms and exacerbations, a large proportion of COPD patients remain untreated. ${ }^{16}$ Furthermore, medication adherence levels for mCOPD medications have been found to be suboptimal and lower than the levels typically observed for treatments for other conditions. ${ }^{5-8}$ One of the reasons for suboptimal adherence to COPD medications may be due to the symptomatic nature of the disease leading to adjustment of medication use depending on how the patients feel on any particular day. ${ }^{9}$ Also, it is expected that COPD patients are prescribed several concurrent therapies since comorbidities are prevalent in these patients and may have an impact on COPD medication adherence. ${ }^{10,11}$ For this study, we hypothesized that adherence to MCOPD medications may be impacted by adherence to medications used to treat chronic conditions other than COPD.

Based on the literature review conducted at the time of this study, we believe our study is the first to explore the impact of overlapping use of non-COPD medications on COPD medication adherence. The results of this study suggest that there is a positive association between nonadherence (or adherence) to mCOPD medications and 
non-adherence (or adherence) to non-COPD medications, ie, patients that are likely to be non-adherent (or adherent) to mCOPD medication are also likely to be non-adherent (or adherent) to non-COPD medications. This finding implies that adherence to medications may be impacted by factors that do not change regardless of disease or medication type but rather is influenced by aspects of the health system (lack of coordinated health care, access restrictions, etc) patient-related considerations (forgetfulness, suboptimal health literacy, etc), and socioeconomic status (education, income etc). ${ }^{13}$

The descriptive analysis results show that adherence for mCOPD medications was low (mean PDC $=0.47$ ) with only $20 \%$ of the study population adherent to $\mathrm{mCOPD}$ medications based on the PDC $\geq 0.80$ threshold. These suboptimal adherence rate results are consistent with previous reports. Toy et $\mathrm{al}^{5}$ reported a PDC of $0.23-0.43$ for inhaled COPD medications, while Neugaard et $\mathrm{al}^{8}$ reported that approximately $30 \%$ of patients were identified as adherent to COPD medications based on PDC $\geq 0.8$ threshold in a Veterans Health Administration patient population.

Several health care payers and providers are focusing their efforts to improve medication adherence through multiple initiatives such as medication therapy management, patient education, and counseling. Since our study findings imply that factors that do not change by medication type may be impacting adherence, more widespread use of patient counseling tools, such as motivational interviewing by pharmacists and other health care providers, may be able to influence patient behavior in improving their adherence irrespective of medication type. ${ }^{17}$

\section{Limitations}

This study has limitations consistent with other retrospective cohort studies conducted using administrative claims databases. First, although statistically significant associations have been shown, causal relationship cannot be determined from the study's findings. Second, COPD medication adherence was assessed based on incident COPD medication use, while prevalent use was evaluated for assessment of nonCOPD medication adherence. This potentially may have had an impact on the associations that were observed in this study. This approach was chosen to enable assessment of overlapping use of COPD and non-COPD medications. Limiting to only new users of non-COPD medications might have severely impacted the sample size. Third, adherence as measured by PDC might not represent true adherence, due to inability to track the use of physician samples and that a prescription claim does not guarantee drug use. Fourth, the study population was a predominantly Medicare population, with an unbalanced geographic distribution in the US. Although 51\% of the medical costs attributable to COPD in the US are paid for by Medicare and almost half of the COPD population is 65 years or older, ${ }^{18,19}$ generalizing the study findings to the entire US population should be approached with caution. Fifth, the total out of pocket costs for medications was not evaluated as a potential driver of medication adherence as it was expected that it would be directly proportional to medication adherence. Lastly, inherent limitations of the administrative claims databases including coding errors and miscoding may have influenced the selection of the study population and result interpretation.

\section{Conclusion}

Adherence to mCOPD medications is low, which was known but verified by these data. Non-adherence (or adherence) to mCOPD medications is positively related to non-adherence (or adherence) to medications for other chronic conditions. This finding implies that adherence to medications may be impacted by factors such as health system-related factors, patient-related factors, and socioeconomic factors that do not change by disease or medication type. Thus, efforts to improve medication adherence should focus more generally on coordination of care, patient education, and patient cost containment.

\section{Disclosure}

The authors report no conflicts of interest in this work.

\section{References}

1. Miniño AM, Xu J, Kochanek KD. Deaths: Preliminary data for 2008. National Vital Statistics Reports. Hyattsville, MD: National Center for Health Statistics; 2010:59(2):1-52.

2. Global Initiative for Chronic Obstructive Lung Disease. Global strategy for diagnosis, management and prevention of COPD; 2015. Available from: http://goldcopd.org/gold-reports/. Accessed October 25, 2016

3. National Institutes of Health, National Heart, Lung, and Blood Institute (NHLBI). Morbidity and Mortality: 2009 Chart Book on Cardiovascular, Lung and Blood Diseases. Bethesda, MD: NHLBI; 2009.

4. Andersson F, Borg S, Jansson SA, et al. The costs of exacerbations in chronic obstructive pulmonary disease (COPD). Respir Med. 2002; 96(9):700-708.

5. Toy EL, Gallagher KF, Stanley EL, Swensen AR, Duh MS. The economic impact of exacerbations of chronic obstructive pulmonary disease and exacerbation definition: a review. COPD. 2010;7(3):214-228.

6. Yeaw J, Benner JS, Walt JG, Sian S, Smith DB. Comparing adherence and persistence across 6 chronic medication classes. J Manag Care Pharm. 2009;15(9):728-740.

7. Cramer JA, Benedict A, Muszbek N, Keskinaslan A, Khan ZM. The significance of compliance and persistence in the treatment of diabetes, hypertension and dyslipidaemia: a review. Int J Clin Pract. 2008;62(1): 76-87. 
8. Neugaard BI, Priest JL, Burch SP, Cantrell CR, Foulis PR. Quality of care for veterans with chronic diseases: performance on quality indicators, medication use and adherence, and health care utilization. Popul Health Manag. 2011;14(2):99-106.

9. George J, Kong DC, Thoman R, Stewart K. Factors associated with medication nonadherence in patients with COPD. Chest. 2005;128(5): 3198-3204.

10. Menzin J, Boulanger L, Marton J, et al. The economic burden of chronic obstructive pulmonary disease (COPD) in a U.S. Medicare population. Respir Med. 2008;102(9):1248-1256.

11. Krigsman K, Moen J, Nilsson JL, Ring L. Refill adherence by the elderly for asthma/chronic obstructive pulmonary disease drugs dispensed over a 10-year period. J Clin Pharm Ther. 2007;32(6):603-611.

12. Ford ES, Murphy LB, Khavjou O, Giles WH, Holt JB, Croft JB. Total and state-specific medical and absenteeism costs of COPD among adults aged $\geq 18$ years in the United States for 2010 and projections through 2020. Chest. 2015;147(1):31-45.

13. World Health Organization. Adherence to long-term therapies: evidence for action. Geneva, Switzerland: World Health Organization; 2003. Available from: http://apps.who.int/iris/bitstream/10665/42682/1/924 1545992.pdf. Accessed March 2, 2016.
14. Leslie RS. Using arrays to calculate medication utilization. In: Proceedings from the 2007 SAS Global Forum; 2007; Orlando, FL. Paper 043-2007. Available from: http://www.lexjansen.com/pharmasug/2008/ pr/pr07.pdf. Accessed November 28, 2016.

15. Farley JF, Harley CR, Devine JW. A comparison of comorbidity measurements to predict healthcare expenditures. Am J Manag Care. 2006; 12(2): $110-119$.

16. Make B, Dutro MP, Paulose-Ram R, Marton JP, Mapel DW. Undertreatment of COPD: a retrospective analysis of US managed care and Medicare patients. Int J Chron Obstruct Pulmon Dis. 2012;7:1-9.

17. Tanzi MG. Counseling to enhance medication adherence. 2012. Available from: https://www.pharmacist.com/counseling-enhancemedication-adherence. Accessed November 28, 2016.

18. Ford ES, Murphy LB, Khavjou O, Giles WH, Holt JB, Croft JB. Total and state-specific medical and absenteeism costs of COPD among adults aged $\geq 18$ years in the United States for 2010 and projections through 2020. Chest. 2015;147(1):31-45.

19. Chronic obstructive pulmonary disease among adults - United States, 2011. Morb Mortal Wkly Rep. 2012;61(46):938-943.

\section{Publish your work in this journal}

The International Journal of COPD is an international, peer-reviewed journal of therapeutics and pharmacology focusing on concise rapid reporting of clinical studies and reviews in COPD. Special focus is given to the pathophysiological processes underlying the disease, intervention programs, patient focused education, and self management protocols.

\section{Dovepress}

This journal is indexed on PubMed Central, MedLine and CAS. The manuscript management system is completely online and includes a very quick and fair peer-review system, which is all easy to use. Visit http://www.dovepress.com/testimonials.php to read real quotes from published authors. 